\title{
Optimal Routing for Decode-Forward in Cooperative Wireless Networks
}

\author{
Lawrence Ong and Mehul Motani
}

\begin{abstract}
We investigate routing in cooperative multipleterminal wireless networks in which the nodes can collaborate with each other in data transmission. First, we motivate cooperation by showing that decode-forward, an informationtheoretic cooperative coding strategy, achieves rates significantly higher than those achievable by the conventional multi-hop routing, a point-to-point non-cooperative coding strategy. We then construct an algorithm to find optimal (rate-maximizing) routes for decode-forward. We show that the algorithm is able to find shortest optimal routes and is optimal in fading channels. However, the algorithm runs in factorial time in the worst case. So, we propose a near-optimal heuristic algorithm that runs in polynomial time. The heuristic algorithm always outputs optimal routes when the nodes transmit independent codewords, and outputs optimal routes with high probability when the nodes transmit arbitrarily correlated codewords. Lastly, we implement decode-forward using low-density parity-check codes to compare the bit error rate performance of different routes.
\end{abstract}

Index Terms-Routing, wireless networks, cooperative systems, information theory, multiuser channels.

\section{INTRODUCTION}

I N AN intriguing paper, Ephremides and Hajek [1] argue that information-theoretic ideas will play an important role in shaping the future of communication networks. In this paper, we study routing (a higher layer communication problem) in wireless networks in which the nodes are capable of interacting and cooperating at the physical layer using information-theoretic cooperative ideas.

Since the wireless medium is broadcast in nature, transmissions of one node can be received by all other nodes listening in the same frequency band. This opens up opportunities for the nodes to cooperate to send information from the source to the destination, e.g., via cooperative relaying [2]-[4] and opportunistic routing [5]. The gain from cooperation has been shown in information-theoretic analyses [6], [7] and demonstrated in practical implementations [8], [9]. In this paper, we focus on the decode-forward coding ${ }^{1}$ strategy (DF) [2][4], an information-theoretic cooperative scheme which has

Paper approved by T.-S. P. Yum, the Editor for Packet Access and Switching of the IEEE Communications Society. Manuscript received March 8, 2009; revised November 3, 2009 and February 19, 2010.

This paper was presented in part at IEEE SECON, San Diego, USA, June 2007, and at IEEE ISIT, Nice, France, June 2007.

L. Ong is with the School of Electrical Engineering and Computer Science, The University of Newcastle, Callaghan, NSW 2308, Australia (e-mail: lawrence.ong@cantab.net).

M. Motani is with the Department of Electrical and Computer Engineering, National University of Singapore, 10 Kent Ridge Crescent, Singapore 119260 (e-mail: motani@nus.edu.sg).

Digital Object Identifier 10.1109/TCOMM.2010.08.090134

${ }^{1}$ The term "coding" is used here to highlight that our approach stems from information theory [10]. been realized with practical Turbo codes [11], [12] and lowdensity parity-check (LDPC) codes [13]-[16] for single-relay networks.

In the cooperative environment, data can be split and transmitted (or repeated using different codes) from multiple nodes to multiple nodes. The traditional notion of a route that focuses on how a data packet travels from the source to the destination fails to capture this complex interaction among the nodes. We propose a new definition for routes that embraces these complex multiple flows of data from the source to the destination. Unfortunately, routing algorithms designed for the conventional non-cooperative multi-hop ( $\mathrm{MH}$ ) routing are no longer optimal (rate-maximizing) in the cooperative framework. In this paper, we propose and analyze routing algorithms for the DF cooperative coding.

\section{A. Contributions}

More specifically, the contributions of this paper are:

(1) We show how much gain one can expect using DF, a cooperative coding strategy, over $\mathrm{MH}$, a non-cooperative coding strategy.

(2) We construct an algorithm that finds optimal (ratemaximizing) routes for DF for single-source single-destination (SSSD) networks. We show that this algorithm can find shortest optimal routes and is optimal in fading channels.

(3) As the above algorithm runs in factorial time in the worst case, we construct a heuristic algorithm, which runs in polynomial time, that always finds optimal routes for DF when the nodes send independent codewords, and finds optimal routes for DF with high probability when the nodes send arbitrarily correlated codewords.

(4) We implement DF using LDPC codes, and compare the bit error rate performance on different routes and with different coding strategies.

\section{B. Related Work}

In the past, routing algorithms were designed specifically for the non-cooperative MH routing strategy. Different metrics for optimization have been studied:

(1) Routes with the lowest cost: A cost is assigned to the link between a node pair. The cost of a route is the sum of the costs of all the links in the route. Bellman-Ford's [17, Section 24.1] and Dijkstra's algorithms [18] find the route with the lowest cost from the source to the destination.

(2) Routes with the least delay: In Ad-hoc On-demand Distance Vector Routing (AODV) [19] and Dynamic Source Routing (DSR) [20], the source node broadcasts a route 
discovery packet. Neighboring nodes receive and re-broadcast the packet. When the destination receives the packet, a route is formed by tracing the path that the packet took.

(3) Opportunistic routes: In Extremely Opportunistic Routing (ExOR) [5], a node broadcasts its data to a set of potential relays. Nodes in this set transmit acknowledgments, and selected nodes forward the data. Though ExOR does not have predefined routes, MH is used on the effective route taken by a packet from the source to the destination.

Routing for the DF cooperative coding has been considered lately in [21], where the authors consider how to minimize the total transmit power of the nodes to achieve a particular rate constraint in the half-duplex wireless network. In this paper, we consider a different metric, i.e., how to maximize the transmission rate for a particular set of power constraints on the nodes in the full-duplex wireless network. In both papers, routing algorithms are proposed for networks with a single source and a single destination, and with perfect synchronization (where coherent reception is possible). While [21] proposes a heuristic algorithm for the static Gaussian channel, both optimal and heuristic algorithms are proposed in this paper. Furthermore, fading channels are also considered in this paper.

In [22], we first studied the routing problem for DF in a class of static wireless networks, where the nodes are constrained to sending independent codewords. In this paper, we extend the result to the general (both static and fading) wireless networks in which the nodes can send arbitrarily correlated codewords.

\section{Organization}

The rest of the paper is organized as follows. In Sec. II, we describe the network model and define what a route means in the cooperative network. We then motivate cooperation by showing that DF achieves significantly higher rates than the single-hop and the MH coding strategies do. We present an optimal routing algorithm for DF in Sec. III and a heuristic near-optimal routing algorithm in Sec. IV. We verify our proposed algorithm on a four-node network in Sec. V, by comparing the achievable rates and the bit error rates of different routes. We conclude the paper in Sec. VI.

\section{Preliminaries}

\section{A. Network Model}

We consider an SSSD network $\mathcal{T}=\{1,2,3 \ldots, T-1, T\}$ with node 1 being the source, node $T$ the destination, and nodes 2 to $(T-1)$ relays. We consider the additive white Gaussian noise channel in which node $t, t=2, \ldots, T$, receives

$$
Y_{t}=\sum_{i=1, \ldots, T-1, i \neq t} \sqrt{\lambda_{i t}} X_{i}+Z_{t}
$$

where $X_{i} \in \mathcal{R}$ is node $i$ 's input to the channel, $Z_{t} \in \mathcal{R}$ is the receiver noise at node $t$, which is an independent zero-mean Gaussian random variable with variance $N_{t}$, and $\lambda_{i t} \in \mathcal{R}^{+}$is the channel gain from node $i$ to node $t$. We assume that node $i$ either transmits at a certain average power $\left(E\left[X_{i}^{2}\right]=P_{i}\right)$ or turns off $\left(X_{i}=0\right)$. We use the standard path loss model for signal propagation: $\lambda_{i j}=\kappa d_{i j}^{-\eta}$, where $d_{i t} \geq 0$ is the distance between nodes $i$ and $t, \eta \geq 2$ is the path loss exponent (with $\eta=2$ for free space transmissions), and $\kappa>0$ is a constant. We define $P_{i t}=\kappa d_{i t}^{-\eta} P_{i}$.

Coherent reception is implicitly assumed in this model, where all transmit signals are received in phase at the receivers. Scenarios without coherent reception are usually studied under the constraint that the nodes transmit independent codewords. This is treated in Theorem 5 in this paper.

We consider full-duplex nodes to gain an understanding of the ultimate limits on transmission rates. We note that achievable rates for any half-duplex network are upper-bounded by its full-duplex counterpart. We also refer the reader to our other work [23] on half-duplex SSSD networks.

Remark 1: We consider single-flow networks as the first step toward understanding more complex networks with multiple flows. The relevance of our work in multiple-flow networks is as follows: (i) In a multiple-flow network where each flow uses an allocated orthogonal channel, the rate of each flow can be optimized in the respective channel using the algorithm derived in this paper. (ii) If we add a new flow in an existing multiple-flow network, the algorithm in this paper finds an optimal route for the new flow. Although adding a new flow might affect existing flows, we can restrict the transmit power of nodes in the new flow to control the amount of interference introduced to the nodes in the existing flows.

\section{B. What is a Route?}

In cooperative wireless networks, data may be transmitted (or repeated using different codes) from multiple nodes to multiple nodes. To describe this collection of multiple paths of information flow from the source to the destination, we re-define a route as follows.

Definition 1: The route taken by a packet from the source to the destination is an ordered set of nodes involved in encoding/transmitting of the packet. The sequence of the nodes in the route is determined by the order in which the nodes' transmit signals first depend on the packet. The destination node is the last node in the route.

It is clear that this definition reduces to the usual notion of a route in the multi-hop case. For the rest of this paper, we denote a route by $\mathcal{M}=\left\{m_{1}, m_{2}, \ldots, m_{|\mathcal{M}|}\right\}$.

\section{Motivating Cooperation}

Now, we present the achievable rates of three coding strategies, namely $\mathrm{DF}, \mathrm{MH}$, and single-hop $(\mathrm{SH})$, using the fournode SSSD network in Fig. 1 as an example for illustration.

1) Single-Hop Coding Strategy (SH): In Fig. 1(a), using $\mathrm{SH}$, the destination only decodes the source's transmissions, not using the relays. For the general $T$-node network, denoting the signal-to-noise ratio (SNR) at the destination (node $T$ ) by $\gamma_{\mathrm{SH}}=\frac{P_{1 T}}{N_{T}}$, the maximum rate achievable using $\mathrm{SH}$ is [24] $R_{\mathrm{SH}}=L\left(\gamma_{\mathrm{SH}}\right)$, where $L(x)=\frac{1}{2} \log (1+x)$. We assume that the nodes that do not participate in relaying the data for a source-destination pair do not cause interference. Throughout this paper, logarithm base 2 is used and hence the unit for rates is bits per channel use. 


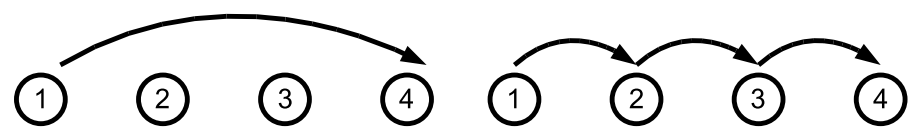

(a) single-hop (b) multi-hop

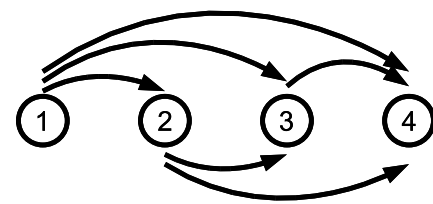

(c) decode-forward

Fig. 1. Different coding strategies for the multiple-terminal network.

2) Multi-Hop Coding Strategy (MH): When nodes 1 and 4 are situated far apart, signals from node 1 are severely attenuated on the way to node 4 . This is when relay nodes 2 and 3 can help. Referring to Fig. 1(b), node 1 transmits to node 2 , which fully decodes the data and re-transmits them to node 3 . Node 3 does the same and relays the data to node 4 .

In $\mathrm{MH}$, the nodes simply transmit packets to the next node in the route, repeating this hop-by-hop until the destination. On a route $\mathcal{M}$ (assuming that $m_{|\mathcal{M}|}=T$ ), as node $m_{t}$ only decodes the transmissions from its immediate upstream node (node $m_{t-1}$ ) and treats all other transmissions as noise, the SNR at node $m_{t}$ is $\gamma_{\mathrm{MH}}\left(m_{t}, \mathcal{M}\right)=$ $\frac{P_{m_{t-1} m_{t}}}{\sum_{a \in \mathcal{M} \backslash\left\{m_{t-1}, m_{t}, T\right\}} P_{a m_{t}}+N_{m_{t}}}$. As all the relays and the destination on the route must fully decode the source message, the overall transmission rate from the source to the destination is $R_{\mathrm{MH}}(\mathcal{M})=\min _{m \in \mathcal{M} \backslash\left\{m_{1}\right\}} L\left(\gamma_{\mathrm{MH}}(m, \mathcal{M})\right)$. We call $R_{\mathrm{MH}}(\mathcal{M})$ the rate supported by the route $\mathcal{M}$ using $\mathrm{MH}$. The maximum rate of $\mathrm{MH}$, optimized over all possible routes, is thus $R_{\mathrm{MH}}^{\max }=$ $\max _{\mathcal{M} \in \Pi(\mathcal{T})} R_{\mathrm{MH}}(\mathcal{M})$, where $\Pi(\mathcal{T})=\left\{\left\{m_{1}, m_{2}, \ldots, m_{|\mathcal{M}|}\right\}\right.$ : $\left.m_{1}=1,\left(m_{2}, \ldots, m_{|\mathcal{M}|-1}\right) \subseteq[2, T-1], m_{|\mathcal{M}|}=T\right\}$, is the set of all possible routes from the source to the destination.

Although we may view relays helping the source to relay data as a form of cooperation, as far as channel coding is concerned, SH and MH still use point-to-point channel codes. We thus categorize them as non-cooperative coding strategies.

3) Decode-Forward Cooperative Coding Strategy (DF): We note that node 3 can hear and decode node 1's transmissions (although they are intended for node 2 in $\mathrm{MH}$ ). This suggests a cooperative coding strategy, depicted in Fig. 1(c), in which each node decodes the transmissions from all upstream nodes, and cancels the interference from downstream nodes, assuming that the downstream nodes decode the source message correctly and forward it.

It has been shown [4] that in order to maximize the DF rate on route $\mathcal{M}$, node $m_{i}, m_{i} \in \mathcal{M} \backslash\left\{m_{|\mathcal{M}|}=\right.$ $T\}$, transmits $X_{m_{i}}=\sum_{j=i+1}^{|\mathcal{M}|} \sqrt{\alpha_{m_{i} m_{j}} P_{m_{i}}} U_{m_{j}}$, for $0 \leq$ $\sum_{j=i+1}^{|\mathcal{M}|} \alpha_{m_{i} m_{j}} \leq 1$ and $\alpha_{m_{i} m_{j}} \geq 0$. Each $U_{m_{j}}$ is an independent Gaussian random variable with unit variance. $\mathcal{A}=\left\{\alpha_{m_{i} m_{j}}: \forall i \in[1,|\mathcal{M}|-1], \forall j \in[i+1,|\mathcal{M}|]\right\}$ is called the set of power splits. $\alpha_{i j}$ is the fraction of the transmit power of node $i$ allocated to transmit the sub-codeletter $U_{j}$. $\mathcal{A}$ controls the correlations among the codewords.

Consider Fig. 1(c) and the DF encoding functions shown in Fig. 2. Let the source messages be $\left\{w_{1}, w_{2}, \ldots\right\}$. In block 1, node 1 sends $w_{1}$ using a fraction $\alpha_{12}$ of its transmit power. Node 2 decodes $w_{1}$ at the end of block 1 . Node 3 overhears the transmission by node 1 and keeps it for further decoding. In block 2 , node 2 forwards $w_{1}$ using a fraction $\alpha_{23}$ of its
Block $1 \quad$ Block $2 \quad$ Block $3 \quad$ Block 4

\begin{tabular}{l|l|l|l|l|}
\cline { 2 - 4 } Node 1 & $\boldsymbol{x}_{1}\left(w_{1}, 0,0\right)$ & $\boldsymbol{x}_{1}\left(w_{2}, w_{1}, 0\right)$ & $\boldsymbol{x}_{1}\left(w_{3}, w_{2}, w_{1}\right)$ & $\boldsymbol{x}_{1}\left(w_{4}, w_{3}, w_{2}\right)$ \\
\cline { 2 - 5 } Node 2 & & $\boldsymbol{x}_{2}\left(w_{1}, 0\right)$ & $\boldsymbol{x}_{2}\left(w_{2}, w_{1}\right)$ & $\boldsymbol{x}_{2}\left(w_{3}, w_{2}\right)$ \\
\cline { 2 - 5 } Node 3 & & & $\boldsymbol{x}_{3}\left(w_{1}\right)$ & $\boldsymbol{x}_{3}\left(w_{2}\right)$ \\
\cline { 2 - 5 } & & &
\end{tabular}

Fig. 2. The encoding of DF, $\boldsymbol{x}_{i}$ denotes the codeword sent by node $i$.

power. In the same block, node 1 sends the new message $w_{2}$ using $\alpha_{12}$ of its power, and helps node 2 to repeat $w_{1}$ using $\alpha_{13}$ of its power, where $0 \leq \alpha_{12}+\alpha_{13} \leq 1$. At the end of block 2 , node 3 combines its received signals in blocks 1 and 2 to decode $w_{1}$. Let us consider the decoding of $w_{1}$ at node 3. The rate contribution from block 1 is $L\left(\frac{\alpha_{12} P_{13}}{N_{3}}\right)$, and the rate contribution from block 2 is $L\left(\frac{\left[\sqrt{\alpha_{13} P_{13}}+\sqrt{\alpha_{23} P_{23}}\right]^{2}}{\alpha_{12} P_{13}+N_{3}}\right)$. Decoding over two blocks, node 3 can decode $w_{1}$ up to the rate $R_{3}=L\left(\frac{\alpha_{12} P_{13}+\left[\sqrt{\alpha_{13} P_{13}}+\sqrt{\alpha_{23} P_{23}}\right]^{2}}{N_{3}}\right)$.

Now, we extend this coding method to the entire route. Consider route $\mathcal{M}$ with power splits $\mathcal{A}$, the SNR at node $m_{t}$ is $\gamma_{\mathrm{DF}}\left(m_{t}, \mathcal{M}, \mathcal{A}\right)=\frac{\sum_{j=2}^{t}\left(\sum_{i=1}^{j-1} \sqrt{\alpha_{m_{i} m_{j}} P_{m_{i} m_{t}}}\right)^{2}}{N_{m_{t}}}$. This means node $m_{t}$ can decode the source message at the rate $R_{m_{t}}(\mathcal{M}, \mathcal{A})=L\left(\gamma_{\mathrm{DF}}\left(m_{t}, \mathcal{M}, \mathcal{A}\right)\right)$. We call this the reception rate of node $m_{t}$.

Again, as all relays must fully decode the source message, DF achieves rates up to $R_{\mathrm{DF}}(\mathcal{M}, \mathcal{A})=$ $\min _{m_{t}}(\mathcal{M}, \mathcal{A})$. Choosing the optimal power splits for the route, DF can achieve rates up to $R_{\mathrm{DF}}(\mathcal{M})=$ $\max _{\mathcal{A}} R_{\mathrm{DF}}(\mathcal{M}, \mathcal{A})$. Similar to $\mathrm{MH}$, we call $R_{\mathrm{DF}}(\mathcal{M})$ the rate supported by route $\mathcal{M}$ using DF. Hence, the maximum DF rate over all possible routes is $R_{\mathrm{DF}}^{\max }=\max _{\mathcal{M} \in \Pi(\mathcal{S})} R_{\mathrm{DF}}(\mathcal{M})$.

4) Comparing the Strategies: If we set $\mathcal{A}^{\prime}=\left\{\alpha_{i, j}\right.$ : $\alpha_{i j}=1$, if $j=i+1$, and $\alpha_{i j}=0$ otherwise $\}$, it is easy to see that for any chosen route $\mathcal{M}$ with four nodes or more, $\gamma_{\mathrm{DF}}(m, \mathcal{M})>\gamma_{\mathrm{MH}}(m, \mathcal{M}), \forall m \in \mathcal{M}$. So, for any $\mathcal{M} \in \Pi(\mathcal{T})$, we have: (i) $R_{\mathrm{DF}}(\mathcal{M})=R_{\mathrm{MH}}(\mathcal{M})=R_{\mathrm{SH}}$, for $|\mathcal{M}|=2$. (ii) $R_{\mathrm{DF}}\left(\mathcal{M}, \mathcal{A}^{\prime}\right) \geq R_{\mathrm{MH}}(\mathcal{M})$, for $|\mathcal{M}|=3$. (iii) $R_{\mathrm{DF}}\left(\mathcal{M}, \mathcal{A}^{\prime}\right)>R_{\mathrm{MH}}(\mathcal{M})$, for $|\mathcal{M}| \geq 4$. Hence, optimizing the power splits for DF, we get $R_{\mathrm{DF}}^{\max } \geq R_{\mathrm{MH}}^{\max } \geq R_{\mathrm{SH}}$.

We now show by numerical calculations how much gain DF gives compared to $\mathrm{MH}$ and SH. Consider a $T$-node SSSD network and fix $d_{1 T}=|\mathcal{M}|-1$. The remaining $(|\mathcal{M}|-2)$ nodes are randomly placed along the straight line joining nodes 1 and $T$. We set $P_{i}=N_{i}=1, \forall i, \kappa=1, \eta=2$. Averaging over randomly generated routes, we see from Fig. 3 that DF achieves rates significantly higher than those by $\mathrm{SH}$ and $\mathrm{MH}$. 


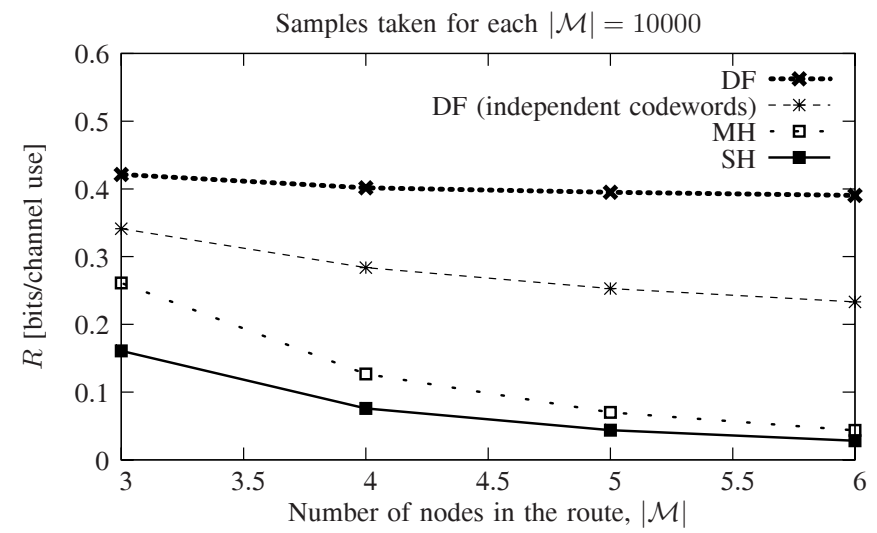

Fig. 3. Comparison of rates achievable by different schemes, with $d_{1 T}=$ $|\mathcal{M}|-1$.

\section{Optimal Routing Algorithms}

\section{A. The Optimal Routing Problem}

We define the optimal route set for DF as $\mathcal{Q}_{\mathrm{DF}} \triangleq\{\mathcal{M} \in$ $\left.\Pi(\mathcal{T}): R_{\mathrm{DF}}(\mathcal{M})=R_{\mathrm{DF}}^{\max }\right\}$. Recall that $\Pi(\mathcal{T})$ is a set of all possible routes from the source to the destination. We define the optimal route set because the rate-maximizing route may not be unique. The optimal DF routing problem is thus to find at least one $\mathcal{M}_{\mathrm{DF}}^{\mathrm{opt}} \in \mathcal{Q}_{\mathrm{DF}}$ and $R_{\mathrm{DF}}\left(\mathcal{M}_{\mathrm{DF}}^{\mathrm{opt}}\right)$. The optimal route set and routing problem for $\mathrm{MH}$ are similarly defined.

Finding optimal routes for $\mathrm{MH}$ and $\mathrm{DF}$ by brute force is hard as the number of routes increases factorially with the number of nodes in the network. Furthermore, finding the DF rate supported by each route involves the optimization of power splits $\mathcal{A}$, whose complexity also grows factorially with the number of nodes. In Section III-B, we will construct an algorithm that finds $\mathcal{M}_{\mathrm{DF}}^{\mathrm{opt}}$, potentially without needing to optimize $\mathcal{A}$.

\section{B. The Nearest Neighbor Set Algorithm}

We start by defining the nearest neighbor set and then present the nearest neighbor set algorithm (NNSA) to find optimal routes for DF.

Definition 2: The nearest neighbor set $\mathcal{N}=$ $\left\{n_{1}, n_{2}, \ldots, n_{|N|}\right\}$ with respect to a route $\mathcal{M}=\left\{m_{1}, m_{2}\right.$, $\left.\ldots, m_{|\mathcal{M}|}\right\}$ is defined as the smallest set $\mathcal{N}$ where each $n \in \mathcal{N} \subseteq \mathcal{T} \backslash \mathcal{M}$ satisfies the condition that $P_{m n} \geq P_{m a}, \quad \forall m \in \mathcal{M}, \forall a \in \mathcal{T} \backslash(\mathcal{M} \cup \mathcal{N})$, with at least one strict inequality for every pair of $(n, a) \in\{(n, a): n \in \mathcal{N}, a \in \mathcal{T} \backslash(\mathcal{M} \cup \mathcal{N})\}$.

Algorithm 1 (NNSA):

1) Initialize $\mathcal{M}=\{1\}$.

2) Find the nearest neighbor set $\mathcal{N}$. The original route $\mathcal{M}$ branches out to $|\mathcal{N}|$ new routes as follows: $\mathcal{M}_{i} \leftarrow \mathcal{M} \cup$ $\left\{n_{i}\right\}, i=1, \ldots,|\mathcal{N}|$.

3) For each new route generated, repeat step 2 until the destination is added to all routes.

When the algorithm terminates, we end up with a set of routes from the source to the destination. We term these routes NNSA candidates, and denote the set of all NNSA candidates by $\mathcal{N} \mathcal{N} \mathcal{S}(\mathcal{T})$. We have shown [25] that any
NNSA candidate that gives the highest supported rate is an optimal route for DF, given in the following theorem.

Theorem 1: Consider an SSSD wireless network. The NNSA candidates that give the highest supported rate are optimal (rate-maximizing) for DF.

To find an optimal route, we first run the NNSA to get $\mathcal{N} \mathcal{N} \mathcal{S} \mathcal{A}(\mathcal{T})$. We then calculate $R_{\mathrm{DF}}(\mathcal{M}), \forall \mathcal{M} \in \mathcal{N} \mathcal{N} \mathcal{S} \mathcal{A}(\mathcal{T})$, and choose the route that gives the highest supported rate. This step requires the optimization of $\mathcal{A}$ for each route, which is out of the scope of this paper. If $|\mathcal{N} \mathcal{N S} \mathcal{A}(\mathcal{T})|=1$, we can find an optimal route without needing to optimize $\mathcal{A}$.

\section{The Performance of the NNSA}

With the NNSA, we only need to search for the optimal route in $\mathcal{N} \mathcal{N} \mathcal{S} \mathcal{A}(\mathcal{T})$, as compared to searching in $\Pi(\mathcal{T})$ using brute force. $|\mathcal{N} \mathcal{N} \mathcal{S} \mathcal{A}(\mathcal{T})|$ determines the number of routes whose rates we need to calculate to find an optimal route. In the worst case scenario, $\mathcal{N} \mathcal{N S \mathcal { A }}(\mathcal{T})=\Pi(\mathcal{T})$. The number of all possible routes is given by all possible selections and permutations of the relays, i.e. $|\Pi(\mathcal{T})|=$ $1+\left(\begin{array}{c}T-2 \\ 1\end{array}\right)+\left(\begin{array}{c}T-2 \\ 2\end{array}\right)+\cdots\left(\begin{array}{c}T-2 \\ T-2\end{array}\right)=O((T-1) !)$, which is factorial in the number of nodes.

Now, we run simulations to obtain the average $|\mathcal{N} \mathcal{N} \mathcal{S} \mathcal{A}(\mathcal{T})|$. For each network size $|\mathcal{T}|$, we randomly generated 10000 networks with nodes randomly and uniformly distributed in a $1 \mathrm{~m} \times 1 \mathrm{~m}$ square area. For each randomly generated network, we ran the NNSA to find the number of NNSA candidates. Half of the time, $|\mathcal{N} \mathcal{N} \mathcal{A}(\mathcal{T})|$ was smaller than $0.715 \%$ of $|\Pi(\mathcal{T})|$ for the 8-node network and smaller than $0.253 \%$ for the 11 -node network. Fig. 4 shows the probability density function of $|\mathcal{N} \mathcal{N} \mathcal{S}(\mathcal{T})|$ for the 11-node network. Fig. 5 shows average $|\mathcal{N} \mathcal{N} \mathcal{S}(\mathcal{T})|$ and $|\Pi(\mathcal{T})|$ for varying number of nodes in the network. The concentration of the pdf of $|\mathcal{N} \mathcal{N} \mathcal{S}(\mathcal{T})|$ on the left end shows that for most of the random networks generated, $|\mathcal{N} \mathcal{N} \mathcal{S} \mathcal{A}(\mathcal{T})|$ is small compared to $|\Pi(\mathcal{T})|$. For the 11-node network, we have the following: (i) Considering the 90-th percentile, we see that $90 \%$ of the time, we can reduce the search space by more than $91 \%$ (compared to brute force). (ii) Considering the 50-th percentile, we see that half of the time, we can reduce the search space by more than $99.3 \%$.

We see that as the number of nodes increases, the number of routes increases exponentially for both the NNSA and brute force. However, on average, we get a 14.6-19.1dB reduction in the number of routes using the NNSA, which is a one to two order of magnitude reduction. Furthermore, the reduction in the number of routes using the NNSA increases as the number of nodes in the network increases.

\section{The Shortest Optimal Route}

Although the best NNSA candidate is an optimal DF route, it is not necessarily the most power-efficient optimal route. In this section, we will further show that shortest optimal routes (defined below) can be obtained from the NNSA.

Definition 3: A shortest optimal route (SOR) is defined as the shortest route that achieves the highest DF rate, i.e., $\mathcal{M}^{\mathrm{SOR}} \in \mathcal{Q}_{\mathrm{DF}}$, s.t. $\left|\mathcal{M}^{\mathrm{SOR}}\right| \leq|\mathcal{M}|, \forall \mathcal{M} \in \mathcal{Q}_{\mathrm{DF}}$. 


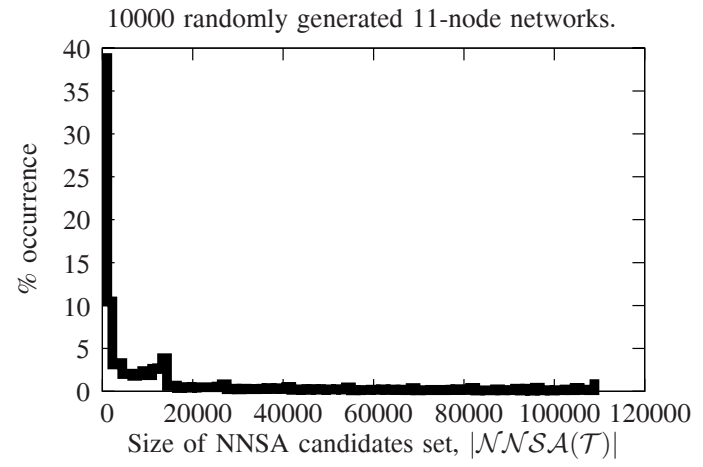

Fig. 4. The pdf of $|\mathcal{N} \mathcal{N} \mathcal{S} \mathcal{A}(\mathcal{T})|$ for the 11-node network, $|\Pi(\mathcal{T})|=$ 986410.

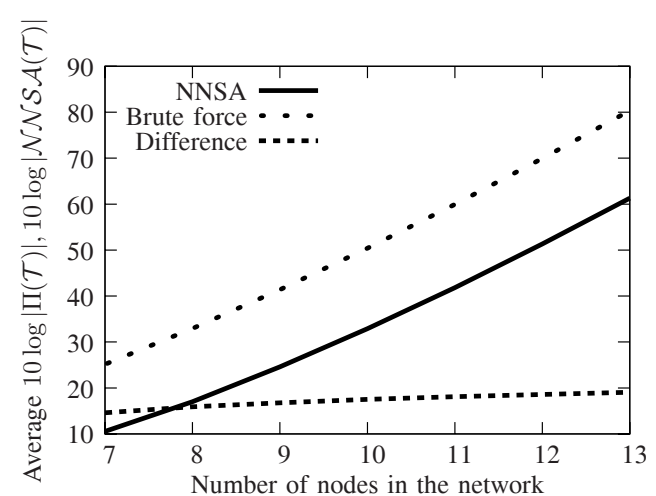

Fig. 5. Average $|\mathcal{N} \mathcal{N} \mathcal{S} \mathcal{A}(\mathcal{T})|$ and $|\Pi(\mathcal{T})|$ for different $|\mathcal{T}|$.

By definition, $R_{\mathrm{DF}}\left(\mathcal{M}^{\mathrm{SOR}}\right)=R_{\mathrm{DF}}^{\max }$. Next, we define a special subset of the NNSA candidates.

Definition 4: The optimal NNSA candidate set $\mathcal{N} \mathcal{N} \mathcal{S A}^{\text {opt }}(\mathcal{T})$ contains all NNSA candidates that achieve the highest rate, meaning, $\mathcal{N} \mathcal{N S} \mathcal{A}^{\text {opt }}(\mathcal{T}) \triangleq\left\{\mathcal{M}^{\prime} \in\right.$

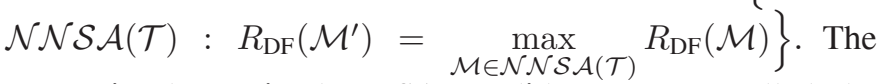
routes in the optimal NNSA candidate set are called the optimal NNSA candidates.

For two routes $\mathcal{A}$ and $\mathcal{B}, \mathcal{A} \subseteq \mathcal{B}$ means $\mathcal{A}$ is a subset of $\mathcal{B}$, and the node order in $\mathcal{A}$ follows that in $\mathcal{B}$. The following theorem says that at least one optimal NNSA candidate contains an SOR.

Theorem 2: One (or more) of the optimal NNSA candidate(s) contains at least an SOR in the correct order, meaning $\exists \mathcal{M}^{\text {SOR }} \subseteq \mathcal{M}$, for some $\mathcal{M} \in \mathcal{N} \mathcal{N S} \mathcal{A}^{\text {opt }}(\mathcal{T})$.

Proof of Theorem 2: See Appendix A.

We now describe an algorithm, the nearest neighbor set pruning algorithm (NNSPA), to find SORs.

Algorithm 2 (NNSPA):

1) Initialize an optimal sub-route set, $\mathcal{O S R} \leftarrow$ $\mathcal{N} \mathcal{N} \mathcal{A}^{\text {opt }}(\mathcal{T})$.

2) For each newly added $\mathcal{M} \in \mathcal{O S R}$, we prune one node from $\left\{m_{2}, \ldots, m_{|\mathcal{M}|-1}\right\}$ at a time to form $(|\mathcal{M}|-2)$ sub-routes.

3) Find the rates supported by all sub-routes. Do the following for all sub-routes:

a) If the sub-route supports rates lower than the optimal rate, discard the sub-route.

b) Else if a sub-route $\mathcal{M}_{s}$ can support the optimal rate, add it into the optimal sub-route set, i.e., $\mathcal{O S R} \leftarrow \mathcal{O S R} \cup \mathcal{M}_{s}$.

4) For each new sub-route formed in step 3b, repeat steps 2-3.

5) Select the shortest route(s) from $\mathcal{O S R}$.

\section{E. The NNSA in Fading Channels}

Now, we investigate the optimality of the NNSA in fading channels, where each $\lambda_{i j}$ in (1) is no longer a constant but is modeled as $\lambda_{i j}=\nu_{i j} \kappa d_{i j}^{-\eta}$, where each $\nu_{i j} \geq 0$ is a random variable. Without loss of generality, we assume $E\left[\nu_{i j}\right]=1, \forall i, j$, i.e., the power of the fading processes is unity. For channels where the fading processes have different power, we can normalize the power to 1 by adjusting $d_{i j}$ accordingly. We assume that the transmitters are unaware of the fading processes and there is no feedback from the receivers about the fading processes. Hence the codewords cannot be chosen as functions of the fading processes $\nu_{i j}$ [26]. We also assume that the receivers have perfect information regarding the fading processes. For fading channels, two measures of "rate" are often used, the ergodic rate and the outage probability, for different applications.

1) Ergodic Rate: When there is no delay constraint for which messages from the source must be decoded at the destination by a certain time, we can use codewords long enough for the fading processes to reflect their ergodic nature [27]. In these delay-tolerant applications, we can achieve ergodic rates averaged over the fading processes.

Since $\nu_{i j}$ are stationary ergodic processes, modifying the results for the point-to-point channel [27], the following ergodic rate is achievable using DF on route $\mathcal{M}$ with power splits $\mathcal{A}$ :

$$
\begin{aligned}
R_{\mathrm{DF}}^{\mathrm{E}}(\mathcal{M}, \mathcal{A}) & =E_{\nu}\left[R_{\mathrm{DF}}(\mathcal{M}, \mathcal{A})\right] \\
& =E_{\nu}\left[\min _{m_{t} \in \mathcal{M} \backslash\{1\}} R_{m_{t}}(\mathcal{M}, \mathcal{A})\right] \\
& =\min _{m_{t} \in \mathcal{M} \backslash\{1\}} R_{m_{t}}^{\mathrm{E}}(\mathcal{M}, \mathcal{A}),
\end{aligned}
$$

where $R_{m_{t}}^{\mathrm{E}}(\mathcal{M}, \mathcal{A})$ is the ergodic reception rate at node $m_{t}$ given by

$$
\begin{aligned}
& R_{m_{t}}^{\mathrm{E}}(\mathcal{M}, \mathcal{A})=E_{\nu}\left[L\left(\gamma_{\mathrm{DF}}\left(m_{t}, \mathcal{M}, \mathcal{A}\right)\right)\right]= \\
& E_{\nu}\left[L\left(\frac{\sum_{j=2}^{t}\left(\sum_{i=1}^{j-1} \sqrt{\alpha_{m_{i} m_{j}} \nu_{m_{i} m_{t}} \kappa d_{m_{i} m_{t}}^{-\eta} P_{m_{i}}}\right)^{2}}{N_{m_{t}}}\right)\right] .
\end{aligned}
$$

We use superscript $E$ to indicate ergodic rate. Eqn. (2c) is because the fading processes are independent. As a result, we can move the expectation operator into the individual reception rate expression in $(2 b)$.

We can view ergodic rate as follows. Consider an instance of the fading processes. The instantaneous reception rate at node $m_{t}$ is $R_{m_{t}}(\mathcal{M}, \mathcal{A})=L\left(\gamma_{\mathrm{DF}}\left(m_{t}, \mathcal{M}, \mathcal{A}\right)\right)$, for some $\nu_{i j} \geq 0$. When the codeword length is long enough, we can achieve the rate averaged over the fading processes in time. Since the fading processes are ergodic, this is equivalent to taking the 
instantaneous rate averaged over the fading ensembles, given by (3a). Applying this to all the nodes in the route, we get (2c). For ergodic rates, we have the following theorem:

Theorem 3: Consider a fading SSSD network $\mathcal{T}$ where the fading processes are i.i.d., stationary, and ergodic. Let the set of NNSA candidates be $\mathcal{N} \mathcal{N} \mathcal{S}(\mathcal{T})$. Then for any power splits $\mathcal{A}$,

$$
\max _{\mathcal{M} \in \mathcal{N} \mathcal{N} \mathcal{S} \mathcal{A}(\mathcal{T})} R_{\mathrm{DF}}^{\mathrm{E}}(\mathcal{M}, \mathcal{A})=\max _{\mathcal{M}^{\prime} \in \Pi(\mathcal{T})} R_{\mathrm{DF}}^{\mathrm{E}}\left(\mathcal{M}^{\prime}, \mathcal{A}\right)
$$

In words, the NNSA candidates that give the highest supported ergodic rate are optimal routes for DF.

Proof of Theorem 3: Let $\mathcal{M}=\left\{m_{1}, \ldots, m_{|\mathcal{M}|}\right\}$ be a route. Assume that the nearest neighbor set is $\mathcal{N}=\left\{a^{*}\right\}$, and let $b \in \mathcal{T} \backslash\left(\mathcal{M} \cup\left\{a^{*}\right\}\right)$ be some non-nearest-neighbor node. Let $\mathcal{M}_{1}=\mathcal{M} \cup\left\{a^{*}\right\}$ and $\mathcal{M}_{2}=\mathcal{M} \cup\{b\}$.

Consider the following fading realizations: $\nu_{i a^{*}}=\nu_{i b}, \forall i \in$ $\mathcal{M}$. From the definition of the nearest neighbor, we must have

$$
\kappa d_{m a^{*}}^{-\eta} P_{m} \geq \kappa d_{m b}^{-\eta} P_{m}, \quad \forall m \in \mathcal{M}, \forall b \in \mathcal{T} \backslash\left(\mathcal{M} \cup\left\{a^{*}\right\}\right) .
$$

So, we have

$$
\begin{aligned}
\nu_{m a^{*}} \kappa d_{m a^{*}}^{-\eta} P_{m} \geq & \nu_{m b} \kappa d_{m b}^{-\eta} P_{m}, \\
& \forall m \in \mathcal{M}, \forall b \in \mathcal{T} \backslash\left(\mathcal{M} \cup\left\{a^{*}\right\}\right) .
\end{aligned}
$$

From [25, Lemma 1], it follows that $R_{\mathrm{DF}}\left(\mathcal{M}_{1}, \mathcal{A}\right) \geq$ $R_{\mathrm{DF}}\left(\mathcal{M}_{2}, \mathcal{A}\right)$ for this fading process realization. Since the fading processes are i.i.d. and ergodic, $p\left(\nu_{i a^{*}}\right)=p\left(\nu_{i b}\right), \forall i \in \mathcal{M}$, averaging over the fading realizations, we get $R_{\mathrm{DF}}^{\mathrm{E}}\left(\mathcal{M}_{1}, \mathcal{A}\right) \geq$ $R_{\mathrm{DF}}^{\mathrm{E}}\left(\mathcal{M}_{2}, \mathcal{A}\right)$.

Using the above trick, i.e., by setting the fading realizations of any two routes in comparison to be equal, and using the same argument as in [25], we can show the optimality of the NNSA for that set of fading realizations. Averaging over the fading realizations, Theorem 3 follows.

Remark 2: In Theorem 3, all we need is that the fading processes are i.i.d. and ergodic. So, Theorem 3 holds for various types of fading models.

2) Supported Rate versus Outage Probability: For delaysensitive applications, the ergodic rates derived in the previous section are no longer applicable. In this section, we consider cases where the fading processes are not ergodic. We model these scenarios by letting all $\nu_{i j}$ be chosen at the beginning of time and be held fixed for all channel uses [28]. This model is also termed quasi-static fading [4]. In this case, for any non-zero transmission rate chosen, there is a non-zero probability that the realization of $\nu_{i j}$ is not able to support it, and the achievable rate in the Shannon sense is zero. So, we investigate the probability that a given transmission rate cannot be supported (using some coding scheme), or supported rate versus outage probability. The definition follows that for the capacity versus outage [29].

Since $\nu_{i j}, \forall i, j$, are random variables, for a given route $\mathcal{M}$ and power splits $\mathcal{A}$, the reception rate at node $m_{t}$ is a random variable. We denote the outage probability at some

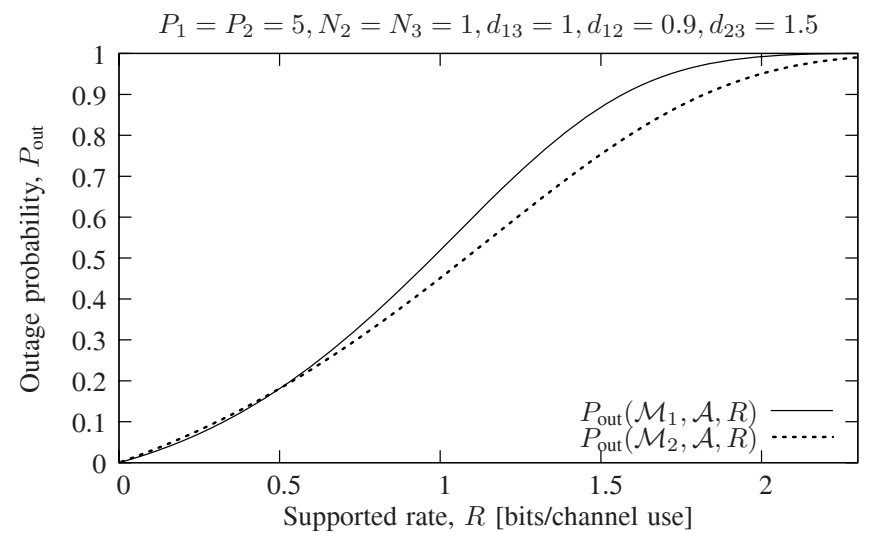

Fig. 6. Supported rate versus outage probability for two routes.

transmission rate $R$ by $P_{\text {out }}(\mathcal{M}, \mathcal{A}, R)$. We note that

$$
\begin{aligned}
& P_{\text {out }}(\mathcal{M}, \mathcal{A}, R) \triangleq \operatorname{Pr}\left(\min _{m_{t} \in \mathcal{M} \backslash\{1\}} R_{m_{t}}(\mathcal{M}, \mathcal{A}) \leq R\right) \\
& =1-\operatorname{Pr}\left(R_{m_{t}}(\mathcal{M}, \mathcal{A})>R, \forall m_{t} \in \mathcal{M} \backslash\{1\}\right) \\
& =1-\prod_{m_{t} \in \mathcal{M} \backslash\{1\}} \operatorname{Pr}\left(R_{m_{t}}(\mathcal{M}, \mathcal{A})>R\right) .
\end{aligned}
$$

In (7c), we assume that the fading processes are independent.

Under quasi-static fading, an optimal route is one which gives the lowest outage probability at all transmission rates. We can show that the NNSA is not always optimal for DF under quasi-static fading, as given by the following theorem.

Theorem 4: Consider a quasi-static fading SSSD network, the NNSA does not always output routes that minimize the outage probability at all rates.

Proof of Theorem 4: Consider a three-node network with Rayleigh fading: $p\left(\nu_{i j}\right)$ is $\frac{1}{\Omega_{i j}} \exp \left(\frac{-\nu_{i j}}{\Omega_{i j}}\right)$ when $\nu_{i j} \geq 0$, and is 0 otherwise, for $i=1,2, j=2,3 . \Omega_{i j}=E\left[\nu_{i j}\right]$ is the fading power. Suppose that the inter-node distances are $d_{12}=0.9, d_{23}=1.5, d_{13}=1$. We consider the following parameters: node 1 and 2 send independent Gaussian codewords (i.e., $\mathcal{A}=\left\{\alpha_{i j}: \alpha_{i j}=1\right.$ if $j=i+1$, and $\alpha_{i j}=$ 0 otherwise $\}$ ) with power $P_{1}=5$ and $P_{2}=5$ respectively, $N_{2}=N_{3}=1, \kappa=1, \eta=2, \Omega_{i j}=1, \forall i, j$. The NNSA route is $\mathcal{M}_{1}=\{1,2,3\}$, and a non-NNSA route is $\mathcal{M}_{2}=\{1,3\}$.

Fig. 6 shows supported rate versus outage probability for $\mathcal{M}_{1}$ and $\mathcal{M}_{2}$. We see that for a large range of $R$, i.e., $0.5<$ $R<2.5, P_{\text {out }}\left(\mathcal{M}_{1}, \mathcal{A}, R\right)>P_{\text {out }}\left(\mathcal{M}_{2}, \mathcal{A}, R\right)$. Hence, the NNSA route does not always give a lower outage probability.

Remark 3: In the above three-node example, we note that the NNSA route $\{1,2,3\}$ gives a higher outage probability (at some supported rates) compared to direct transmission $\{1,3\}$ because we need to ensure that this rate is supported at each relay and the destination. Hence, when we consider the supported rate versus outage probability, the route length plays an important part. A longer route is likely to result in a higher outage probability. 
TABLE I

PERFORMANCE OF THE MSPA

\begin{tabular}{|c||c|c|}
\hline$T$ & Average $\frac{R_{\mathrm{DF}}\left(\mathcal{M}_{\mathrm{MSPA}}\right)}{R_{\mathrm{DF}}^{\max }}$ & Fraction of optimal routes using MSPA \\
\hline 3 & 1 & 1 \\
4 & 0.9999950 & 0.99882 \\
5 & 0.9999513 & 0.99522 \\
6 & 0.9999399 & 0.99194 \\
\hline
\end{tabular}

\section{A Heuristic Algorithm}

\section{A. The Maximum Sum-of-Received-Power Algorithm}

Using the NNSA, a route is constructed by adding the "nexthop" node from the nearest neighbor set to the partial route. If the nearest neighbor set contains multiple node, the current route branches out to multiple routes, leading to a potentially large NNSA candidate sets.

To avoid this, we consider a heuristic approach that starts from the source node and repeatedly adds only one "good" candidate from the nearest neighbor set until the destination is reached. For the choice of the next-hop node, we consider the node that receives the largest sum of received power from all the nodes in the existing route. We call this the maximum sum-of-received-power algorithm (MSPA). By adding only one node to the partial route, we prevent the algorithm from branching out to multiple routes. This heuristic approach yields only one route, regardless of the network size. We now explicitly describe the MSPA.

Algorithm 3 (MSPA):

1) Initialize $\mathcal{M}=\{1\}$.

2) For every node $t \in \mathcal{T} \backslash \mathcal{M}$, find the sum of received power from all nodes in $\mathcal{M}$ to $t$, i.e., $\sum_{i \in \mathcal{M}} P_{i t}$.

3) Let $a^{*}$ be any node with the highest sum of received power, i.e., $\sum_{i \in \mathcal{M}} P_{i a^{*}} \geq \sum_{j \in \mathcal{M}} P_{j t}, \forall t \in \mathcal{T} \backslash \mathcal{M}$. Append node $a^{*}$ to the route: $\mathcal{M} \leftarrow \mathcal{M} \cup\left\{a^{*}\right\}$.

4) Repeat steps 2-3 until the destination is added to the route.

Remark 4: Assuming that the value of the previous sumof-received-power computations are cached, the complexity of step 2 in MSPA is $O(T)$ because there are at most $(T-1)$ nodes not in the route. The complexity of the comparisons in step 3 is $O(T)$. Steps $2-3$ are repeated at most $(T-1)$ times, giving a worst case complexity of the MSPA of $O\left(T^{2}\right)$.

We have the following two theorems for the MSPA:

Theorem 5 ([22]): In an SSSD wireless network in which the nodes send independent codewords, the MSPA route is optimal (rate-maximizing) for DF.

Theorem 6: In an SSSD wireless network in which the nodes send can send arbitrarily correlated codewords, the MSPA route might not be optimal (rate-maximizing) for DF.

Proof of Theorem 6: (By contradiction) Consider a four-node network with node coordinates $1(0,0), 2(0.418,0)$, $3(0.209,0.6755)$, and 4(0.995,0). Assume $P_{i}=1, N_{i}=1, \kappa=$ $1, \eta=2$. The MSPA route is $\mathcal{M}_{1}=\{1,2,4\}$. The NNSA outputs $\mathcal{M}_{1}$ and $\mathcal{M}_{2}=\{1,2,3,4\}$. It is easy to compute that $R_{\mathrm{DF}}\left(\mathcal{M}_{1}\right)=1.30826$ and $R_{\mathrm{DF}}\left(\mathcal{M}_{2}\right)=1.31576$.

\section{B. The Performance of the MSPA}

We now investigate how well the MSPA route, $\mathcal{M}_{\mathrm{MSPA}}$, performs compared to the optimal route in SSSD networks.

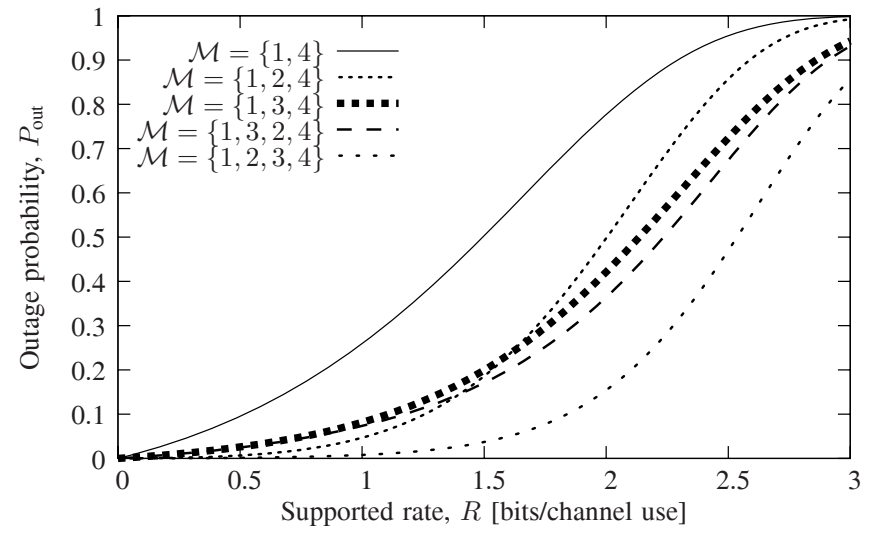

Fig. 7. Supported rate versus outage probability.

Due to the complexity involved in optimizing the power splits, we only simulate networks up to 6 nodes, i.e., $T \leq 6$. For each $T$, we randomly place $T$ nodes in a $(T-1) \mathrm{m} \times(T-1) \mathrm{m}$ square area. The source and the destination are randomly chosen. We run the NNSA to find the optimal rate $R_{\mathrm{DF}}^{\max }$, and run the MSPA to find $R_{\mathrm{DF}}\left(\mathcal{M}_{\mathrm{MSPA}}\right)$. The results are shown in Table I. With high probability, the MSPA is able to find an optimal route. Also, $R_{\mathrm{DF}}\left(\mathcal{M}_{\mathrm{MSPA}}\right)$ is a good indicator of $R_{\mathrm{DF}}^{\max }$.

\section{Numerical Computations And Simulations}

\section{A. Network Topology}

In this section, we compare the performance of different routes in a four-node network with node coordinates $1(0,0.5)$, $2(0,0.4), 3(0,1)$, and $4(0,1.5)$. We set the following parameters: $\kappa=1, \eta=2, P_{i}=10, \forall i$, and $N_{j}=1, \forall j$.

\section{B. Performance in Static Channels}

Using the NNSA, we obtain the optimal route $\mathcal{M}^{*}=$ $\{1,2,3,4\}$. We can verify that this is the optimal route by comparing the achievable rates of the different routes as follows: $R_{\mathrm{DF}}(\{1,4\})=1.7297, R_{\mathrm{DF}}(\{1,2,4\})=2.1339$, $R_{\mathrm{DF}}(\{1,3,4\})=2.6788, R_{\mathrm{DF}}(\{1,2,3,4\})=2.9445$, $R_{\mathrm{DF}}(\{1,3,2,4\})=2.6788$.

\section{Performance in Fading Channels}

Fig. 7 shows supported rates versus outage probabilities of the five possible routes in the network. We assume that all fading processes are independent Rayleigh with $\Omega_{i j}=1, \forall i, j$, and the nodes send independent codewords. We see that the NNSA route, i.e., the optimal route, has the lowest outage probability across all rates.

\section{Performance with LDPC Codes}

In the previous section, we computed achievable rates based on an information-theoretic approach. In this section, we compare different strategies and routes using low-density parity-check (LDPC) codes [30], [31] with rate-compatible puncturing [32]. For codes of finite length, there are bound to be decoding errors, and a common metric for comparison is the bit error rate (BER). A route with a lower BER is better than one with a higher BER.

Remark 5: We do not perform code optimization here. One might be able to find other codes which achieve lower BERs. 
1) Channel Model: Consider a point-to-point continuous time channel, where the receivers are subject to white Gaussian noise with noise power spectral density $N_{0}$. Assume that node $i$ transmits using BPSK with power $P_{i}$, and the transmit symbol duration is 1 unit time, $T_{c}=1$. After performing matched filtering at node $j$ at every unit time, the output is $y_{j}=\sum_{i \neq j} \sqrt{\kappa d_{i j}^{-\eta}} x_{i}+z_{j}$, where $x_{i}= \pm \sqrt{P_{i} T_{c}}= \pm \sqrt{E_{c}}$ is a binary input into the channel. $E_{c}=E_{b} R_{\mathrm{LDPC}}$, where $E_{b}$ is the energy per uncoded bit and $R_{\mathrm{LDPC}}$ is the rate of the punctured LDPC code, which is the ratio of uncoded bits to the transmitted coded bits. $z_{j}$ is zero-mean independent Gaussian noise with variance $N_{j}=N_{0} / 2$. Note that the transmit symbol duration is 1 unit time. So, the energy per uncoded message bit per noise power spectral density is $E_{b} / N_{0}=P_{i} /\left(2 N_{j} R_{\mathrm{LDPC}}\right)$.

2) Code Construction: Consider an $(n, k)$ LDPC code. The encoder takes in a $k$-bit message $\boldsymbol{m} \in\{0,1\}^{k}$ and outputs an $n$-bit codeword $\boldsymbol{c}(\boldsymbol{m})=\left[c_{1} \cdots c_{n}\right] \in\{0,1\}^{n}$. The rate of this unpunctured code is thus $k / n$. The decoder takes in received signal $\boldsymbol{y} \in \mathcal{R}^{n}$ and output a message estimate $\hat{\boldsymbol{m}}=g(\boldsymbol{y}) \in$ $\{0,1\}^{k}$.

To implement DF, rate-compatible punctured [32] LDPC codes are used. For an LDPC code, the puncturing algorithm in [32] outputs a sequence of indices. When we want to transmit at a higher code rate (i.e., using a fewer number of transmitted codebits), we puncture the codebits according to the indices output by the algorithm. For example, say the puncturing algorithm outputs $\left[\phi_{1}, \ldots, \phi_{n}\right], \phi_{i} \in\{1, \ldots, n\}$, $\phi_{i} \neq \phi_{j}$ if $i \neq j$. We define the re-arranged codeword to be $h(\boldsymbol{c})=\left[c_{\phi_{1}} \cdots c_{\phi_{n}}\right]$. To transmit at rate $R_{\mathrm{LDPC}}=k / n^{\prime}$, where $n^{\prime} \leq n$, we only transmit $\left[c_{\phi_{1}} \cdots c_{\phi_{n^{\prime}}}\right]$. We term $\left[c_{1} \cdots c_{n}\right]$ the mother (unpunctured) code and $\left[c_{\phi_{1}} \cdots c_{\phi_{n^{\prime}}}\right]$ the punctured code.

Now, we extend this puncturing idea to LDPC for the multiple-terminal network. Let us give an example of LDPC with puncturing and incremental redundancy on route $\{1,2,4\}$ using a $(n, k)$ mother code. We denote $h(\boldsymbol{c})=\left[\boldsymbol{c}_{\alpha} \boldsymbol{c}_{\beta}\right]$, where $\boldsymbol{c}_{\alpha}=\left[c_{\phi_{1}} \cdots c_{\phi_{n / 2}}\right]$ and $\boldsymbol{c}_{\beta}=\left[c_{\phi_{(n / 2)+1}} \cdots c_{\phi_{n}}\right]$. In block 1 , node 1 transmits only $n / 2$ bits, i.e., $\boldsymbol{x}_{1}\left(\boldsymbol{m}_{1}\right)=$ $2 \sqrt{E_{c}}\left(\boldsymbol{c}_{\alpha}\left(\boldsymbol{m}_{1}\right)-0.5\right)$. At the end of block 1, node 2 receives the noisy $n / 2$ bits, $\boldsymbol{y}_{2,1}$. It pads the punctured $n / 2$ bits with zeros, re-arranges the signals, and feeds them into the decoder to estimate $\hat{\boldsymbol{m}}_{1}=g\left(h^{-1}\left(\left[\boldsymbol{y}_{2,1} \mathbf{0}\right]\right)\right)$. In block 1 , node 4 also receives the noisy $n / 2$ bits. Instead of decoding now, it keeps its received signals $\boldsymbol{y}_{4,1}$ for decoding in block 2. In block 2, node 1 sends new message, $\boldsymbol{x}_{1}\left(\boldsymbol{m}_{2}\right)=2 \sqrt{E_{c}}\left(\boldsymbol{c}_{\alpha}\left(\boldsymbol{m}_{2}\right)-0.5\right)$; and node 2 sends the "missing" $n / 2$ bits from the codeword of the old message, $\boldsymbol{x}_{2}\left(\hat{\boldsymbol{m}}_{1}\right)=2 \sqrt{E_{c}}\left(\boldsymbol{c}_{\beta}\left(\hat{\boldsymbol{m}}_{1}\right)-0.5\right)$. Node 4 combines its received signals, i.e., $\boldsymbol{y}_{4,1}$ in block 1 and $\boldsymbol{y}_{4,2}$ in block 2, to decode $\hat{\boldsymbol{m}}_{1}=g\left(h^{-1}\left(\left[\boldsymbol{y}_{4,1} \boldsymbol{y}_{4,2}\right]\right)\right)$. Here, we see how node 4 combines its received signals over two blocks, from nodes 1 and 2 , to decode a message. The rate of this code is $R_{\mathrm{LDPC}}=k /(n / 2)=2 k / n$.

3) BER Simulation Results: We simulate transmissions on different routes in the four-node network described in Section V-A using a $R_{\mathrm{LDPC}}=1 / 2$ code punctured from a rate-1/6 mother LDPC code. From Fig. 8 , we see that: (i) For a given route, DF performs better than $\mathrm{MH}$. (ii) Routing backward can help in DF but not in MH. (3) The NNSA

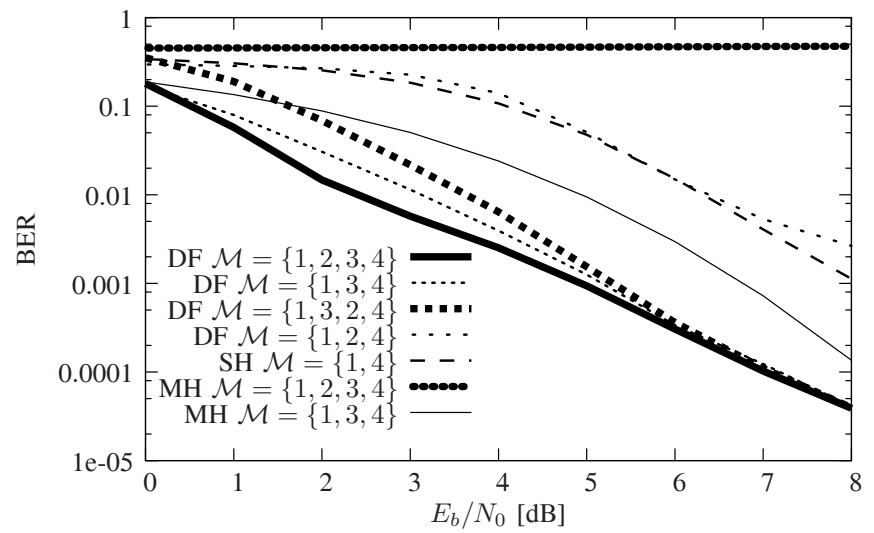

Fig. 8. Information bit error rate (BER) versus $E_{b} / N_{0}$ of various codes and routes.

route (which is also the MSPA route), $\{1,2,3,4\}$, gives the best performance (lowest BER) compared to other routes using DF.

Remark 6: We use an $R_{\mathrm{LDPC}}=1 / 2$ code punctured from a rate-1/6 mother LDPC code for all routes in the comparison, including direct transmission, route $\{1,4\}$. An alternative is to use a rate- $1 / 2$ unpunctured LDPC code. We argue that choosing the former gives practical advantages. We demonstrate this point with the following example.

Example 1: Consider an $R_{\mathrm{LDPC}}=1 / 2$ code punctured from a rate- $1 / 6$ mother LDPC code, and consider a chosen route $\{1,2,4\}$ in a fading channel. Node 1 sends $n / 3$ of the coded bits. Node 2 decodes the source message and sends another $n / 3$ of the coded bits. Supposed that the channels $1 \rightarrow 4$ and $2 \rightarrow 4$ experience a deep fade, and node 4 is not able to decode the source message from the $2 n / 3$ bits sent by nodes 1 and 2 . If another node, node 3 , that over-hears these transmission, is able to decode the source message, it can help to send the remaining $n / 3$ of the coded bits to node 4 . With these extra codebits, node 4 might be able to decode the source message. Node 4 does not need to change its decoding algorithm when it decodes these extra bits from node 3 .

Remark 7: From a practical view point, there are concerns in implementing DF-based codes in large networks. First, a node decodes from all upstream nodes (e.g., the destination decodes signals from the source and all relays). Depending on the topology and the environment, signals at the receiver may be severely attenuated and difficult to be detected. Second, large networks may be distributed over a wide area, and synchronizing all the nodes' transmissions for DF implementation is difficult. One possible solution to these practical issues is to use myopic DF where cooperation is done locally [6].

\section{CONCLUding Remarks}

In this paper, we investigated routing in networks in which the nodes are capable of information-theoretic cooperation. We first showed that the cooperative decode-forward (DF) strategy gives a significant gain in transmission rate over the traditional multi-hop $(\mathrm{MH})$ strategy. We then proposed the nearest neighbor set algorithm (NNSA) to find DF optimal routes, that is, routes which maximize the rates achievable by DF, and showed the following: (i) The size of the NNSA 
candidate set can be significantly smaller than the set of all routes, reducing the search space by one to two orders of magnitude compared to brute force search over all possible routes. (ii) The NNSA can be extended to find the shortest optimal route, one that achieves the highest rate with the lowest resource consumption. (iii) The NNSA is optimal in fading channels in the sense that an NNSA route maximizes the ergodic rate. However, the NNSA is not guaranteed to find routes that minimizes the outage probability.

The NNSA has a worst case running time which is equal to brute force search, i.e., factorial in the number of nodes in the network. In view of this, we designed a near-optimal, polynomial-running-time, heuristic - the maximum sum-ofreceived-power algorithm (MSPA), which always finds an optimal route when the nodes send independent codewords and finds an optimal route with high probability when the nodes send arbitrarily correlated codewords.

Finally, we explored how one might use DF-based cooperation in practice. We implemented DF using punctured LDPC codes with incremental redundancy and compared the BER performance of different routes. As expected, the NNSA route achieved the lowest BER. Unexpectedly, the best DF route required backward routing away from the destination.

This work explores connections between the fields of communications, networking, and information theory. The direct connection is the impact of cooperative transmission on network routing. In most routing algorithms, a route progresses in the direction toward the destination. We found that with DF cooperation, routing away from the destination can lead to higher rates and better performance, an observation which is supported by network simulations using LDPC codes. There are several other directions for future work, including how to scale information theoretic cooperation to large networks and discovering other connections among the three fields.

\section{APPENDIX A}

\section{PROOF OF THEOREM 2}

First, we show that an SOR is contained in at least one of the NNSA candidates.

Lemma 1: If $\mathcal{M}=\left\{m_{1}^{*}, m_{2}^{*}, \ldots, m_{|\mathcal{M}|-1}^{*}, m_{|\mathcal{M}|}^{*}\right\}$ is an SOR, then $P_{a m_{|\mathcal{M}|-1}^{*}} \geq P_{a m_{|\mathcal{M}|}^{*}}$ for at least one $a \in$ $\left\{m_{1}^{*}, m_{2}^{*}, \ldots, m_{|\mathcal{M}|-2}^{*}\right\}$.

Proof of Lemma 1: (By contradiction) If $P_{a m_{|\mathcal{M}|-1}^{*}}<$ $P_{a m_{|\mathcal{M}|}^{*}}, \forall a \in\left\{m_{1}^{*}, m_{2}^{*}, \ldots, m_{|\mathcal{M}|-2}^{*}\right\}$, then $R_{\mathrm{DF}}\left(\mathcal{M}_{1}, \mathcal{A}\right) \geq$ $R_{\mathrm{DF}}(\mathcal{M}, \mathcal{A})$, for any $\mathcal{A}$ where $\mathcal{M}_{1}=\left\{m_{1}^{*}, m_{2}^{*}, \ldots\right.$, $\left.m_{|\mathcal{M}|-2}^{*}, m_{|\mathcal{M}|}^{*}\right\}$. So, $\mathcal{M}_{1}$ is a shorter route and can support rates as high as $\mathcal{M}$ does. So, the latter cannot be an SOR.

Lemma 1 says that the second last node must not receive a lower SNR from each of the first $(|\mathcal{M}|-2)$ nodes in the route than the last node does. It does not mean that the second last node must be the from the nearest neighbor set with respect to the first $(|\mathcal{M}|-2)$ nodes. However, when the partial route is $\left\{m_{1}^{*}, \ldots, m_{|\mathcal{M}|-2}^{*}\right\}$, the NNSA will eventually include $m_{|\mathcal{M}|-1}^{*}$ in one or more of the NNSA candidates before it includes $m_{|\mathcal{M}|}^{*}$.

We now extend Lemma 1 to the third last node. Using a similar argument, we can show that $P_{a m_{|\mathcal{M}|-2}^{*}} \geq P_{a m_{|\mathcal{M}|}^{*}}$, for some $a \in\left\{m_{1}^{*}, m_{2}^{*}, \ldots, m_{|\mathcal{M}|-3}^{*}\right\}$. It then follows that if the partial route is $\left\{m_{1}^{*}, \ldots, m_{|\mathcal{M}|-3}^{*}\right\}$, the NNSA will definitely include $m_{|\mathcal{M}|-2}^{*}$ in one or more NNSA candidates before it includes $m_{|\mathcal{M}|}^{*}$.

We have shown that the NNSA will include $m_{|\mathcal{M}|-2}^{*}$ and $m_{|\mathcal{M}|-1}^{*}$ before $m_{|\mathcal{M}|}^{*}$ in one or more NNSA candidates. This does not guarantee that there exists an NNSA candidate that includes $\left(m_{|\mathcal{M}|-2}^{*}, m_{|\mathcal{M}|-1}^{*}, m_{|\mathcal{M}|}^{*}\right)$ in that order. For instance, if $P_{a m_{|\mathcal{M}|-1}^{*}}>P_{a m_{|\mathcal{M}|-2}^{*}}, \forall a \in$ $\left\{m_{1}^{*}, \ldots, m_{|\mathcal{M}|-3}^{*}\right\}$, then none of the NNSA candidates will contain $\mathcal{M}$ with the correct order, but some will contain $\mathcal{M}^{\prime}=\left\{m_{1}^{*}, m_{2}^{*}, \ldots, m_{|\mathcal{M}|-3}^{*}, m_{|\mathcal{M}|-1}^{*}, m_{|\mathcal{M}|-2}^{*}, m_{|\mathcal{M}|}^{*}\right\}$. However, in this case, $R_{\mathrm{DF}}\left(\mathcal{M}^{\prime}\right) \geq R_{\mathrm{DF}}(\mathcal{M})$, and hence $\mathcal{M}^{\prime}$ is also an SOR. Extending the above arguments to all the nodes in an SOR, we have the following lemma.

Lemma 2: One or more NNSA candidates contain an SOR, with the correct order. This means if $\mathcal{M}=$ $\left\{m_{1}^{*}, m_{2}^{*}, \ldots, m_{|\mathcal{M}|-1}^{*}, m_{|\mathcal{M}|}^{*}\right\}$ is the SOR contained in one of the NNSA candidates, $m_{2}^{*}$ comes after $m_{1}^{*}$ in the NNSA candidate, and $m_{3}^{*}$ comes after $m_{2}^{*}$, and so on. But there might be other nodes in between them. For example, the NNSA candidate might be $\left\{m_{1}^{*}, \ldots, m_{|\mathcal{M}|-2}^{*}, a, m_{|\mathcal{M}|-1}^{*}, m_{|\mathcal{M}|}^{*}\right\}$.

Now, we further prove that an SOR is contained in one or more optimal NNSA candidates, not just NNSA candidates.

$$
\begin{gathered}
\text { Consider } \\
\left.m_{1}^{*}, m_{2}^{*}, \ldots, m_{K}^{*}, m_{K+1}^{*}, \ldots, m_{|\mathcal{M}|-1}^{*}, m_{|\mathcal{M}|}^{*}\right\}
\end{gathered},
$$

and an NNSA candidate $\mathcal{M}_{1}$

$\left\{m_{1}^{*}, m_{2}^{*}, \ldots, m_{K}^{*}, a, m_{K+1}^{*}, \ldots, m_{|\mathcal{M}|-1}^{*}, m_{|\mathcal{M}|}^{*}\right\}$. This is possible since $\mathcal{M}_{1}$ contains $\mathcal{M}^{*}$ in the correct order.

We denote the set of the first $K$ nodes as $\mathcal{M}_{1}^{\prime}$, i.e., $\mathcal{M}_{1}^{\prime}=\left\{m_{1}^{*}, \ldots, m_{K}^{*}\right\}$. Since $\mathcal{M}_{1}$ is formed by the NNSA algorithm, a must be from the nearest neighbor set with respect to $\mathcal{M}_{1}^{\prime}$. Next, we consider three cases: (1) $m_{K+1}^{*}$ is not from the nearest neighbor set with respect to $\mathcal{M}_{1}^{\prime}$. (2) $\left(a, m_{K+1}^{*}, m_{K+2}^{*}, \ldots, m_{K+i}^{*}\right)$ are from the nearest neighbor set with respect to $\mathcal{M}_{1}^{\prime}$, and $m_{K+i}^{*} \neq m_{|\mathcal{M}|}^{*}$. (3) $\left(a, m_{K+1}^{*}, m_{K+2}^{*}, \ldots, m_{M}^{*}\right)$ are from the nearest neighbor set with respect to $\mathcal{M}_{1}^{\prime}$.

Consider case (1). The condition means that $P_{b a}>$ $P_{b m_{K+1}^{*}}, \forall b \in \mathcal{M}_{1}^{\prime}$. Using the set of $\left\{\alpha_{i j}^{*}\right\}$ that maximizes $\mathcal{M}^{*}$, and setting any arbitrary power splits $\left\{\alpha_{a j}\right\}$ for node $a$, we have: (i) $R_{m_{n}^{*}}\left(\mathcal{M}_{1}\right)=R_{m_{n}^{*}}\left(\mathcal{M}^{*}\right)$, for $n=2,3, \ldots, K$. (ii) $R_{a}\left(\mathcal{M}_{1}\right) \geq R_{m_{K+1}^{*}}\left(\mathcal{M}^{*}\right)$. (iii) $R_{m_{n}^{*}}\left(\mathcal{M}_{1}\right) \geq$ $R_{m_{n}^{*}}\left(\mathcal{M}^{*}\right)$, for $n=K+1, K+2, \ldots,|\mathcal{M}|$. Hence $R_{\mathrm{DF}}\left(\mathcal{M}_{1}\right) \geq R_{\mathrm{DF}}\left(\mathcal{M}^{*}\right)=R_{\mathrm{DF}}^{\max }$, and $\mathcal{M}_{1}$ must be from $\mathcal{N} \mathcal{N} \mathcal{S} \mathcal{A}^{\text {opt }}(\mathcal{T})$.

Consider case (2). Since $\left(a, m_{K+1}^{*}, m_{K+2}^{*}, \ldots, m_{K+i}^{*}\right)$ are from the nearest neighbor set with respect to $\mathcal{M}_{1}^{\prime}$, it follows that $\mathcal{M}_{2}=$ $\left\{m_{1}^{*}, m_{2}^{*}, \ldots, m_{K}^{*}, m_{K+1}^{*}, \ldots, m_{K+i}^{*}, a, m_{K+i+1}^{*}, \ldots, m_{M}^{*}\right\}$ is also an NNSA candidate. This is because the NNSA includes all selections and permutations of the nodes in the nearest neighbor set. Now, consider $\mathcal{M}_{2}^{\prime}=\left\{m_{1}^{*}, m_{2}^{*}, \ldots, m_{K}^{*}\right.$, $\left.m_{K+1}^{*}, \ldots, m_{K+i}^{*}\right\}$, and:

- If $P_{b a}>P_{b m_{K+i+1}^{*}}, \forall b \in \mathcal{M}_{2}^{\prime}$, meaning $a$ is the only nearest neighbor to $\mathcal{M}_{2}^{\prime}$, then using the same argument for case (1) above, we can show that $R_{\mathrm{DF}}\left(\mathcal{M}_{2}\right) \geq R_{\mathrm{DF}}\left(\mathcal{M}^{*}\right)=$ $R_{\mathrm{DF}}^{\max }$, and $\mathcal{M}_{2}$ must be from $\mathcal{N} \mathcal{N} \mathcal{S} \mathcal{A}^{\text {opt }}(\mathcal{T})$. This means the SOR $\mathcal{M}^{*}$ is contained in another optimal NNSA candidate $\mathcal{M}_{2}$. 
- Else if $\left(a, m_{K+i+1}^{*}, m_{K+i+j}^{*}\right)$ are the nearest neighbors with respect to $\mathcal{M}_{2}^{\prime}$, we rearrange and notice that $\mathcal{M}_{3}=\left\{m_{1}^{*}, m_{2}^{*}, \ldots, m_{K+i+j}^{*}, a, m_{K+i+j+1}^{*}, \ldots, m_{M}^{*}\right\}$ is also an NNSA candidate. We stop when node $a$ is the only nearest neighbor. This is equivalent to case (1). Else, we continue to "push" node $a$ backward until $a$ is in the nearest neighbor set that includes the destination, which is case (3).

Now, consider case (3). The nodes $\left(a, m_{K+1}^{*}, m_{K+2}^{*}, \ldots, m_{M}^{*}\right)$ are from the nearest neighbor set with respect to $\mathcal{M}_{1}^{\prime}$. Since the NNSA includes all possible selections and permutations of nodes in the nearest neighbor set, $\mathcal{M}^{*}$ is an NNSA candidate. Since it achieves $R_{\mathrm{DF}}^{\max }$, it is an optimal NNSA candidate.

We have shown that an SOR $\mathcal{M}^{*}$ must be contained in some NNSA candidate $\mathcal{M}_{1}$. If $\mathcal{M}_{1}$ has more than one nodes compared to $\mathcal{M}^{*}$, we can use the above method to "push" the extra nodes backward in the route and re-arrange the nodes until they are the nearest neighbor set with respect to the upstream nodes (or the extra nodes and all their downstream nodes are the nearest neighbor set with respect to all upstream nodes, which means that $\mathcal{M}^{*}$ is an optimal NNSA candidate). Let $\mathcal{M}_{2}$ be this new route. We showed that $\mathcal{M}_{2}$ is also an NNSA candidate. As the rate supported by $\mathcal{M}_{2}$ cannot be lower than the SOR, $\mathcal{M}_{2}$ is an optimal NNSA candidate and it contains $\mathcal{M}^{*}$. Hence, we have Theorem 2.

\section{REFERENCES}

[1] A. Ephremides and B. Hajek, "Information theory and communication networks: an unconsummated union," IEEE Trans. Inf. Theory, vol. 44, no. 6, pp. 2416-2434, Oct. 1998.

[2] T. M. Cover and A. A. El Gamal, "Capacity theorems for the relay channel," IEEE Trans. Inf. Theory, vol. IT-25, no. 5, pp. 572-584, Sep. 1979.

[3] L. Xie and P. R. Kumar, "An achievable rate for the multiple level relay channel," IEEE Trans. Inf. Theory, vol. 51, no. 4, pp. 1348-1358, Apr. 2005.

[4] G. Kramer, M. Gastpar, and P. Gupta, "Cooperative strategies and capacity theorems for relay networks," IEEE Trans. Inf. Theory, vol. 51, no. 9 , pp. 3037-3063, Sep. 2005.

[5] S. Biswas and R. Morris, "Opportunistic routing in multi-hop wireless networks," SIGCOMM Comput. Commun. Rev., vol. 34, no. 1, pp. 69-74, 2004.

[6] L. Ong and M. Motani, "Myopic coding in multiterminal networks," IEEE Trans. Inf. Theory, vol. 54, no. 7, pp. 3295-3314, July 2008.

[7] A. Sendonaris, E. Erkip, and B. Aazhang, "User cooperation diversitypart I: system description," IEEE Trans. Commun., vol. 51, no. 11, pp. 1927-1938, Nov. 2003

[8] —, "User cooperation diversity_part II: implementation aspects and performance analysis," IEEE Trans. Inf. Theory, vol. 51, no. 11, pp. 1939-1948, Nov. 2003

[9] T. L. Lim, V. Srivastava, and M. Motani, "Selective cooperation based on link distance estimations in wireless ad-hoc networks," in Proc. IEEE Int. Symp. World Wireless, Mobile Multimedia Netw. (WOWMOM), Niagara-Falls, Buffalo, NY, June 2006, pp. 391-400.

[10] T. M. Cover and J. A. Thomas, Elements of Information Theory. WileyInterscience, 1991.

[11] B. Zhao and M. C. Valenti, "Distributed turbo coded diversity for relay channel," Electron. Lett., vol. 39, no. 10, pp. 786-787, May 2003.

[12] Z. Zhang, I. Bahceci, and T. M. Duman, "Capacity approaching codes for relay channels," in Proc. IEEE Int. Symp. Inf. Theory (ISIT), Chicago, IL, June 2004, p. 2.

[13] M. A. Khojastepour, N. Ahmed, and B. Aazhang, "Code design for the relay channel and factor graph decoding," in Proc. 38th Annual Asilomar Conf. Signals, Syst., Comput., Pacific Grove, CA, Nov. 2004, pp. 2000-2004.

[14] A. Chakrabarti, A. De-Baynast, A. Sabharwal, and B. Aazhang, "Low density parity check codes for the relay channel," IEEE J. Sel. Areas Commun., vol. 25, no. 2, pp. 280-291, Feb. 2007.
[15] J. Ezri and M. Gastpar, "On the performance of independently designed ldpc codes for the relay channel," in Proc. IEEE Int. Symp. Inf. Theory (ISIT), Seattle, WA, July 2006, pp. 977-981.

[16] P. Razaghi and W. Yu, "Bilayer LDPC codes for the relay channel," in Proc. IEEE Int. Conf. Commun. (ICC), Istanbul, Turkey, June 2006, pp. 1574-1579.

[17] T. H. Cormen, C. E. Leiserson, R. L. Rivest, and C. Stein, Introduction to Algorithms. MIT Press and McGraw-Hill, 2001.

[18] E. W. Dijkstra, "A note on two problems in connection with graphs," Numerische Math., vol. 1, pp. 269-271, 1959.

[19] C. E. Perkins, E. M. Belding-Royer, and S. Das, July 2003, "Ad hoc on demand distance vector (aodv) routing. IETF RFC 3561." [Online]. Available: http://moment.cs.ucsb.edu/pub/rfc3561.txt

[20] D. B. Johnson and D. Maltz, "Dynamic source routing in ad hoc wireless networks," in Mobile Computing. Kluwer Academic Publishers, 1996, vol. 353.

[21] A. E. Khandani, J. Abounadi, E. Modiano, and L. Zheng, "Cooperative routing in static wireless networks," IEEE Trans. Commun., vol. 55, no. 11 , pp. 2185-2192, Nov. 2007.

[22] L. Ong and M. Motani, "Optimal routing for decode-and-forward based cooperation in wireless networks," in Proc. 4th Annu. IEEE Conf. Sensor Ad Hoc Commun. Netw. (SECON), San Diego, CA, June 2007, pp. 334343.

[23] L. Ong, W. Wang, and M. Motani, "Achievable rates and optimal schedules for half duplex multiple-relay networks," in Proc. 46th Allerton Conf. Commun., Control, Comput., Monticello, IL, Sept. 2008, pp. 1317 1324.

[24] C. E. Shannon, "A mathematical theory of communication," Bell Syst. Tech. J., vol. 27, pp. 379-423, 623-656, July-Oct. 1948.

[25] L. Ong and M. Motani, "Optimal routing for the decode-and-forward strategy in the Gaussian multiple relay channel," in Proc. IEEE Int. Symp. Inf. Theory (ISIT), Nice, France, June 2007, pp. 1061-1065.

[26] D. N. C. Tse and S. V. Hanly, "Multiaccess fading channels-part I: polymatroid structure, optimal resource allocation and throughput capacities," IEEE Trans. Inf. Theory, vol. 44, no. 7, pp. 2796-2815, Nov. 1998.

[27] E. Biglieri, J. Proakis, and S. Shamai, "Fading channels: informationtheoretic and communications aspects," IEEE Trans. Inf. Theory, vol. 44, no. 6, pp. 2619-2692, Oct. 1998.

[28] E. Telatar, "Capacity of the multiple antenna Gaussian channel," Europ. Trans. Telecommun., vol. 10, no. 6, pp. 585-595, Nov.-Dec. 1999.

[29] L. H. Ozarow, S. Shamai, and A. D. Wyner, "Information theoretic considerations for cellular mobile radio," IEEE Trans. Veh. Technol. pp. 359-378, May 1994.

[30] R. G. Gallager, "Low density parity check codes," IRE Trans. Inf. Theory, vol. IT-8, pp. 21-28, Jan. 1962.

[31] D. J. C. MacKay, "Good error-correcting codes based on very sparse matrices," IEEE Trans. Inf. Theory, vol. 45, no. 2, pp. 399-430, Mar. 1999.

[32] J. Ha, J. Kim, D. Klinc, and S. W. McLaughlin, "Rate-compatible punctured low-density parity-check codes with short block lengths," IEEE Trans. Inf. Theory, vol. 52, no. 2, pp. 728-738, Feb. 2006.

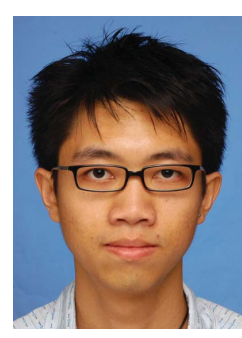

Lawrence Ong is currently a post-doctoral researcher at the School of Electrical Engineering and Computer Science at The University of Newcastle. He received the BEng degree in electrical engineering from the Department of Electrical and Computer Engineering (ECE) at National University of Singapore (NUS), Singapore, in 2001. After graduation from NUS, he worked in a telecommunication company in Singapore from June 2001 to August 2002. His main task was software management, planning, and integration of the mobile switching network. He then pursued and received the MPhil degree from University of Cambridge, Cambridge, UK, during 2002-2004, working on recipe representation, interpretation, product resource communication, and negotiation mechanisms. After receiving the MPhil degree, he enrolled at NUS for the $\mathrm{PhD}$ program and worked on information theoretic approaches to understanding wireless networks. He received the Ph.D. degree from the Department of ECE at NUS in July 2008. He was a research fellow at the Department of ECE at NUS from June 2007 to August 2008. 


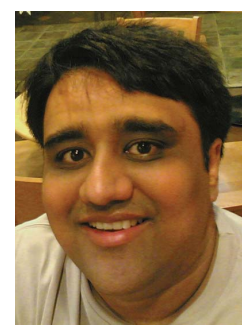

Mehul Motani is an Associate Professor in the Electrical and Computer Engineering Department at the National University of Singapore. Previously, he was a Research Scientist at the Institute for Infocomm Research in Singapore for three years and a Systems Engineer at Lockheed Martin in Syracuse, NY for over four years. He graduated with a Ph.D. from Cornell University, focusing on information theory and coding for CDMA systems.

Recently he has been working on research problems which sit at the boundary of information theory, communications and networking, including the design of wireless ad-hoc and sensor network systems.

He was awarded the Intel Foundation Fellowship for work related to his Ph.D. He has served on the organizing committees of ISIT, WiNC and ICCS, and the technical program committees of MobiCom, Infocom, ICNP, SECON, and several other conferences. He participates actively in IEEE and ACM and has served as the secretary of the IEEE Information Theory Society Board of Governors. He is currently an Associate Editor for the IEEE Transactions on Information Theory. 